\title{
Ferroelastic domain walls as templates for multiferroic devices
}

Cite as: J. Appl. Phys. 128, 164104 (2020); https://doi.org/10.1063/5.0029160

Submitted: 10 September 2020 . Accepted: 09 October 2020 . Published Online: 28 October 2020

Ekhard K. H. Salje (i)
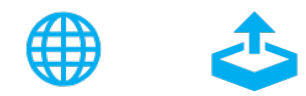

View Online

Export Citation

CrossMark

Meet the Next Generation of Quantum Analyzers And Join the Launch Event on November 17th

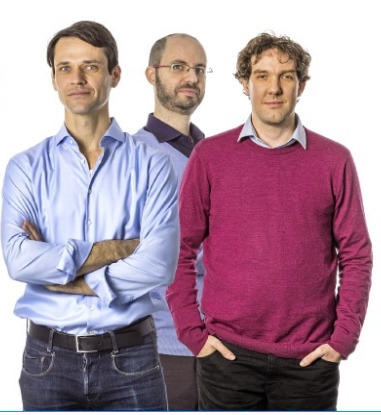

Zurich

Instruments 


\title{
Ferroelastic domain walls as templates for multiferroic devices
}

\author{
Cite as: J. Appl. Phys. 128, 164104 (2020); doi: 10.1063/5.0029160 \\ Submitted: 10 September 2020 . Accepted: 9 October 2020 . \\ Published Online: 28 October 2020
}

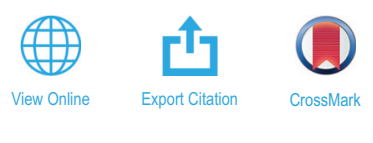

Ekhard K. H. Salje ${ }^{\text {a) (D }}$

\author{
AFFILIATIONS \\ Department of Earth Sciences, University of Cambridge, Downing Street, Cambridge CB2 3EQ, United Kingdom
}

Note: This paper is part of the Special Topic on Domains and Domain Walls in Ferroic Materials

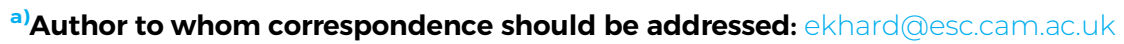

\begin{abstract}
Ferroelastic domain walls are typically twin interfaces. They contain a multitude of emerging properties, including (super-)conductivity, polarity, optically activity, and photovoltaic properties and may contain a number of magnetic properties even when there is no magnetic element in the ferroelastic matrix. Current progress to determine these properties is reviewed. Local wall properties change a little if the domain walls are deformed, but external fields can shift the location of the wall. Mobile domain boundaries carry with them the locus of any emerging property. The double flexibility of functional domain walls and the possibility to move domain boundaries provide a new design concept for device manufacturing. Some potential avenues for device designs are discussed.
\end{abstract}

Published under license by AIP Publishing. https://doi.org/10.1063/5.0029160

\section{INTRODUCTION}

Current research in ferroelastics is dominated by the investigation of emerging properties of domain walls (Salje, 2012) rather than the properties of the bulk. Most domain walls in this research were simple twin walls and were already explored in the 1990s (Lawless and Fousek, 1970 and Janovec et al., 1999). The simple geometrical and crystallographic descriptions of twin walls were summarized by Salje (1993) and put into connection with ferroelasticity. A massive change in the perception of twin boundaries emerged when two new fundamental aspects were identified over the last few decades. First twin walls in some materials show (super-)conductivity (Aird and Salje, 1998; Seidel et al., 2009; 2010; Guyonnet et al., 2011; Farokhipoor and Noheda, 2011; Meier et al., 2012; Rojac et al., 2017; Sluka et al., 2013; and Ma et al., 2018), polarity (Goncalves-Ferreira et al., 2008; Scott et al., 2012; Salje et al., 2013; Wei et al., 2014; Nataf et al., 2017; Van Aert et al., 2012; Salje, 2010; Catalan et al., 2012; Seidel, 2012; and Meier, 2015), optical frequency doubling in confined spaces (Yokota et al., 2017; 2018; 2019), phonon shifts (Salje, 1992), and photovoltaics (e.g., Yang and Alexe, 2020). Second, external forces move domain walls unless they are highly pinned. These movements control spatially and temporarily emerging properties. The combination of local properties and the ability to shift their locus often leads to the formation of complex domain patterns that are at the heart of domain boundary engineering (Salje et al., 1983; Salje, 2010; Salje and Parlinkski, 1991; Salje and Wruck, 1983; Kim et al., 2010; and Viehland and Salje, 2014). The goal is now to use these design options to develop novel device materials, which perform better than current bulk devices.

Increased spatial resolution of atomic force microscopy and high-resolution transmission electron microscopy and developments in optical techniques (Nataf and Guennou, 2020) have helped to identify active sites in domain walls (Kirbus et al., 2019 and Eng et al., 1999) and clarified that the thickness of the nonmagnetic domain walls are typically in the range of $1-10 \mathrm{~nm}$ (Chrosch and Salje, 1999; Hayward et al., 1996; Van Aert et al., 2012; Gonnisen et al., 2016; Jiang et al., 2018; and Shilo et al., 2004). In this paper, we progress one step further and focus on the dynamic properties of domain walls. Under external forcing, their dynamics can be divided into "smooth" and "non-smooth" movements. Smooth movements during ferroelectric and ferroelastic switching are traditionally described by the nucleation and growth (respectively shrinkage) of domains (Ishibashi and Takagi, 1971 and Miller, 1958). Non-smooth, collective movements operate differently. Domain walls exhibit jumps, which sometimes trigger other jumps and create avalanches. The domain patterns are fractal (Zaiser, 2006) and defy the mechanical continuums description (Lee et al., 2006; Salje and Ishibashi, 1996; Salje, 1988; and Conti et al., 2011). Their temporal evolution defines avalanches that are 
described within the framework of "crackling noise" (Sethna et al., 2001 and Salje and Dahmen, 2014) where the movements of domain walls are characterized statistically by analyzing the probability $\mathrm{P}(\mathrm{J})$ of a jump $\mathrm{J}$ to occur. It turns out that the probability is very often power law distributed with $\mathrm{P}(\mathrm{J}) \sim \mathrm{J}^{-\alpha} \cdot \mathrm{F}(\mathrm{J})$, where $\mathrm{F}(\mathrm{J})$ describes an exponential and non-universal cutoff. The exponent $\alpha$ is typically related to universality classes, whereby the pinningdepinning scenario is dominant for ferroic materials. Experimentally, a large number of experimental techniques (acoustic emission, depolarization current measurements, optical microscopy, dynamical mechanical analyzer, etc.) have been applied to observe these jumps and their distributions (Vives et al., 1994; Gallardo et al., 2010; and Nataf et al., 2014). Considerable theoretical work has also been undertaken in order to provide the tools to analyze these distributions, many of which are based on the maximum-likelihood (ML) method (Alava et al., 2006; Clauset et al., 2009; Baró and Vives, 2012; Salje and Dahmen, 2014; and Salje et al., 2017a; 2017b). In addition, jerky non-smooth movements can be overlaid by smooth wall progressions so that one needs to distinguish between jerks (non-smooth) and less jerky behavior. More recently, Weiss was the first to use the terms "wild" (for jerky, non-smooth) and "mild" (for smooth) behaviors. This intuitive notion correctly implies that the smooth behavior also contains highly non-linear excitations and is not confined to harmonic phonon processes (Weiss et al., 2015; Yang et al., 2020; Chen et al., 2020; and Zhang et al., 2020).

\section{PINNING, AVALANCHES, AND THEIR MEASUREMENTS}

Domain walls are highly mobile at $\mathrm{T}>400 \mathrm{~K}$ in the prototypic ferroelastic material $\mathrm{LaAlO}_{3}$, which we now use to exemplify the approach (Hayward et al., 2011). The wall width increases with temperature and shows a typical Landau anomaly at the phase transition temperature at $813 \mathrm{~K}$ (Chrosch and Salje, 1999; and Hayward et al., 2005). The lateral wall movement (Harrison and Salje, 2010; 2011; Puchberger et al., 2017; and Kustov et al., 2018) proceeds by pinning/depinning movements or the formation of local tweed structures (Salje et al., 2016). The domain walls are polar (Yokota et al., 2018), although the polarity is weaker than in most other known ferroelastic materials (Yokota et al., 2020a; 2020b; 2019; 2014). A typical scenario for the wall movement is shown in Fig. 1. Here, a domain delaminates from the top surface and forms a bent interface between the two adjacent ferroelastic domains. The bending sequence is typical for the elastic response of the system and is characterized by the Larkin length (i.e., the length of the wiggle) of the interface. Local pinning equally bends the interface. The shapes of interfaces pinned by defects, surface pinning, or junction pinning for elastically anisotropic ferroelastics were derived in continuums theory by Salje and Ishibashi (1996) and compared with experimental observations by Salje et al. (1998).

The movement of the walls under slowly increasing stress leads to a combination of smooth movements (wall sliding without pinning) and jerky events when walls first pin and then suddenly de-pin after a local threshold stress is overcome. The wall sliding is well described by the time dependent Landau-Ginzburg theory in the viscous limit (Salje, 2012). The statistical analysis of jerk events yields a power law distribution (Harrison and Salje, 2010). The energy exponent is close to $\epsilon=1.6$ in close agreement with predictions of the mean field theory (Salje and Dahmen, 2014). The smooth sliding can carry emerging properties such as electric dipole moments through the sample and is hence at the core of memory elements where polar walls carry information in close analogy to the magnetic racetrack memory technology (Parkin et al., 2008) as described below. The reading of the energy proceeds either electrostatically via the deviation of electric currents in a thin

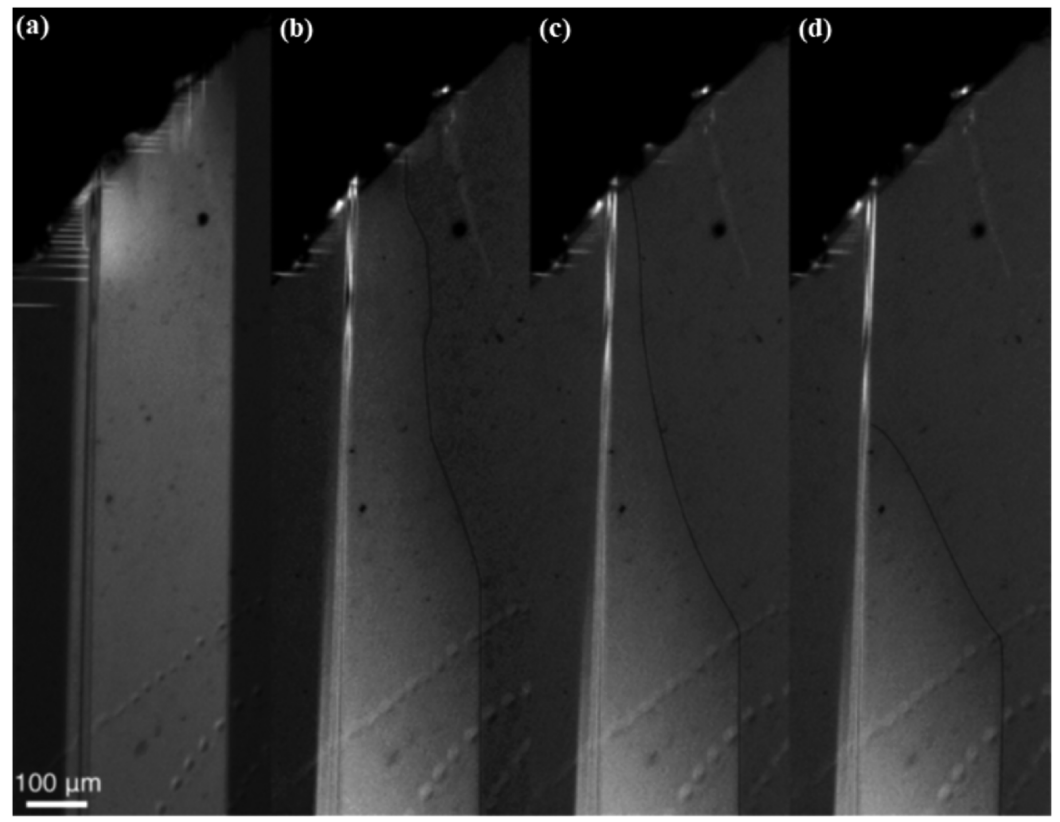

FIG. 1. Optical image of a ferroelastic twin wall in $\mathrm{LaAlO}_{3}$ attached to the upper sample surface (left). Reproduced from Harrison and Salje, Appl. Phys. Lett. 99, 151915 (2011). Copyright 2011 AIP Publishing LLC. One domain appears lighter and the other domain appears darker. The domain boundary in (a) is the sample surface seen under an oblique angle. The lighter domain is then pulled down by an elastic force. An upper domain wall nucleates [(b) and (c)] and detaches under increasing external strain (d). The newly created twin wall is bent first and then straightens after a long relaxation time. Each spontaneous change of the line profile is "jerky" and gives rise to acoustic emission by the sample. 
layer of $\mathrm{SrTiO}_{3}$ that is deposited on top of the $\mathrm{LaAlO}_{3}$ sample (Frenkel et al., 2017) or magnetically as demonstrated by Fontcuberta et al. (2015). These authors showed that dramatic changes of the magnetic domain structure of a neighboring magnetic layer $\left(\mathrm{La}_{0.5} \mathrm{Sr}_{0.5} \mathrm{MnO}_{3}\right)$, which is epitaxially clamped on a ferroelastic substrate, reproduces very well the domain configuration of the ferroelastic domain.

A novel magnetic coupling between domain wall motion and displacement vortices was reported by Lu et al. (2020) where moving ferroelastic needle domains or comb domains generate polarization vortices. These vortices generate displacement currents because the moving atoms are charged. This is the origin of magnetic moments perpendicular to the plane of the vortex. The calculated changes of the domain patterns generate strong mechanical forces if the needle movements are sufficiently fast and generate large displacement currents. The magnetic moment can reach several tens of the Bohr magnetons, which is an estimate for a thin film of $\sim 100$ lattice planes and movements of the vortex at the speed of sound. The predicted magnetic fields found in thin slab are much larger than those observed experimentally in $\mathrm{SrTiO}_{3} /$ $\mathrm{LaAlO}_{3}$ heterostructures (Christensen et al., 2019), which may be due to weaker forcing by slow changes of the domain patterns under their experimental conditions. These results show that dynamic ferroelastic domain patterns can be observed by other experimentally accessible parameters besides the ferroelastic strain as described in detail by Catalan et al. (2012). They appear in polar electric measurements, optical experiments like in the second harmonic generation (SHG), magnetically in magnetic cover layers, or, perhaps most importantly and previously unknown, magnetically by vortex formation and the induced circular displacement current near the domain walls, even when there is no magnetic element present in the material or the wall.

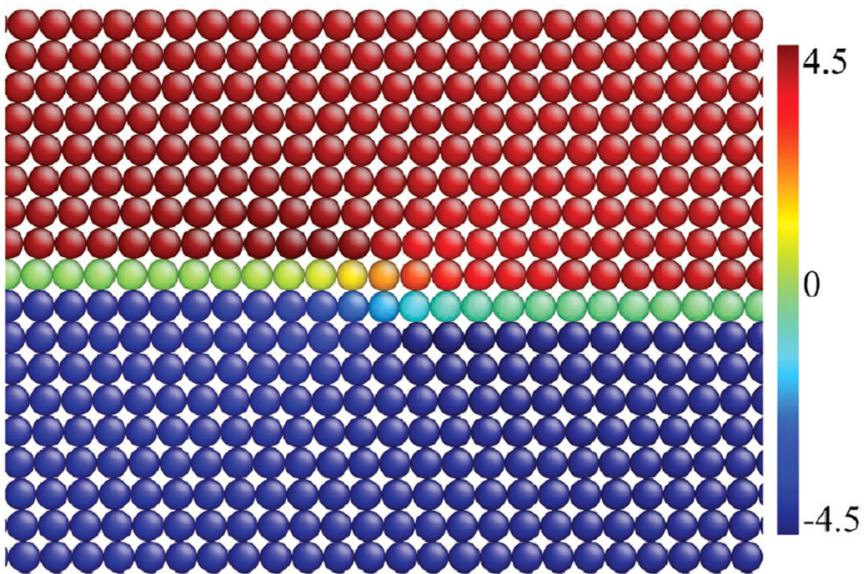

(a)
Jerky movement may partially destroy the smooth wall movements and add noise to the memory readout (Salje et al., 2019). The noise signal is, within cutoffs, scale invariant and will extend to rare but extremely strong events. Furthermore, the time intervals between jerks, the so-called "waiting times," are also power law distributed with exponents near unity for high frequencies. This implies that to filter jerks electronically is very challenging at high $(\mathrm{THz})$ frequencies. Jerks due to pinning by extrinsic defects can be partially avoided in extremely pure materials. Avoiding intrinsic topological defects requires that no junctions occur between twin walls, which can be achieved for parallel wall configurations in narrow thin films, nano-pillars, or nano-wires (Yang et al., 2016).

\section{WALLS IN WALLS: FAST KINK MOVEMENTS}

Some avalanches are intrinsic to twin walls and cannot be avoided. The most prominent examples are captured by the concept of "walls in walls and domains in domains" (Salje et al., 2013 and Scott et al., 2012). In such a scenario, a twin wall nucleates kinks inside the twin wall. Such kinks are extremely common when samples are bent (Emelyanov et al., 2001 and Pertsev et al., 2000) and also nucleate under external forcing during the sliding motion of the walls. Note that the same movement occurs in polytypic materials where different polytypic stackings transform among each other by nucleation of kinks (Salje et al., 1987). The jerk amplitudes of kink motions are much smaller than during pinning-depinning events and are often overlooked when measured in acoustic emission experiments. A typical computer simulated example is shown in Fig. 2 (Salje et al., 2017).

Moving kinks emit shear waves (Fig. 3) showing typical ultrasonic cones at high speeds. In contrast to studies of dislocations (Gumbsch and Gao, 1999 and Jin et al., 2006), speeds well above

FIG. 2. A kink nucleates inside a twin wall when external shear stress is applied (a) and emits a strain wave in the typical Eshelby pattern (b). Reproduced from Salje et al., Adv. Funct. Mater. 27, 1700367 (2017). Copyright 2017 Wiley. The color bar in (a) indicates the vertical shear angle (i.e., the ferroelasic shear) between $4.5^{\circ}$ and $-4.5^{\circ}$. The color bar shows the horizontal shear angle (secondary shear). 


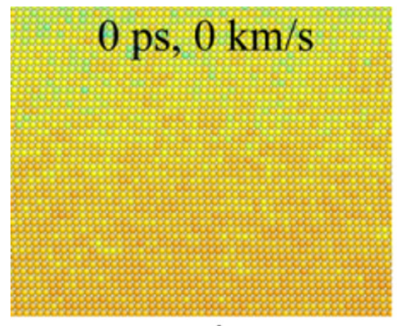

static

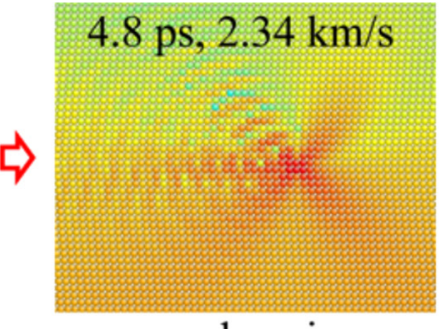

subsonic

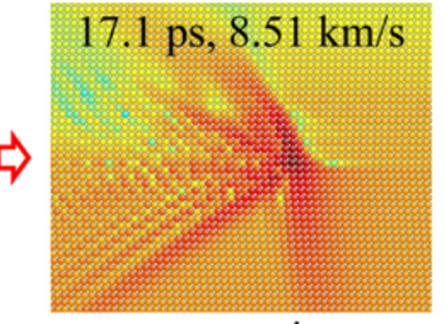

transonic

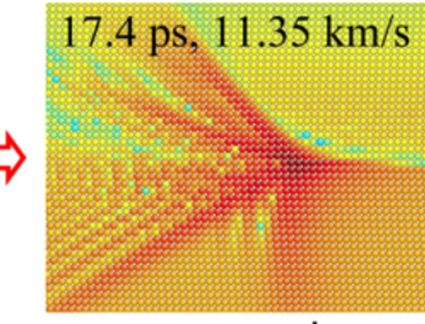

supersonic

FIG. 3. Further shear accelerates the kink moving at speeds in excess of the speed of sound after Salje et al., Adv. Funct. Mater. 27, 1700367 (2017). Copyright 2017 Wiley. The colors indicate the horizontal shear deformation, which evolves from a nearly uniform distribution for the static kink to dynamic strain waves carried by phonons and emitted by the moving kink.

the upper sonic velocity are attainable without suppression by gap phenomena. The kinks equally carry electric and magnetic fingerprints (Lu et al., 2019a; 2019b) and are potentially information carriers in their own right. Reversing the direction of forcing leads to de-acceleration and reversal of the propagation direction. Equivalent oscillatory movements are observed in resonant ultrasonic spectroscopy (RUS) experiments (Carpenter, 2015).

Non-linear strain interactions lead to dynamic singularities. The most common event appears to be the mother-daughter nucleation where moving kinks spontaneously split into kinks and anti-kinks traveling in opposite directions (Salje et al., 2017). The movement of a first generation kink with speed $v$ splits into two movements with kink speed $\delta v$ for the daughter kink and $v-\delta v$ for the mother kink. The mother kink slows by the speed $\delta v$ of the daughter event and conserves momentum. The statistical significance of these mother-daughter events is still unknown.

\section{FERROELECTRIC AVALANCHES DURING SWITCHING}

The first full avalanche analysis of scale invariant switching in $\mathrm{BaTiO}_{3}$ was undertaken by Salje et al. (2019).
During several switching cycles (Figs. 4 and 5), the sample emits acoustic noise, which is measured using commercial piezoelectric sensors (Vallen Ltd).

The maximum acoustic activity appears near the coercive field with lower activities spread over the entire hysteresis loop. Analysis of the AE spectra (Salje et al., 2019) shows the characteristic power law distribution of the jerk energies and all dependent quantities.

The validity of the power law extends over $\sim 6$ orders of magnitude and is limited by the dynamic range of the detection system and not by the physical mechanism of the switching process. The probability functions with such high resolution are extremely hard to measure, and these data are exceptional, so far, for switching of ferroelectric materials. The quality of the dataset made it possible to test all statistical correlations, which are experimentally accessible (Salje et al., 2019). The energy exponent over the hysteresis is 1.66 (Fig. 6) while a criticality with $\epsilon=1.33$ appears at the coercive field (equivalent to the critical point in phase transitions) (Salje and Dahmen, 2014 and Casals et al., unpublished). This is in close agreement with the predictions of the mean field theory (Friedman et al., 2012). Other parameters ( jerk energies, amplitudes, waiting times, avalanche durations, etc.) equally confirm the mean field character of the switching process. The direct observation of the domain movements by optical microscopy became possible in 2019

Acoustic Emission

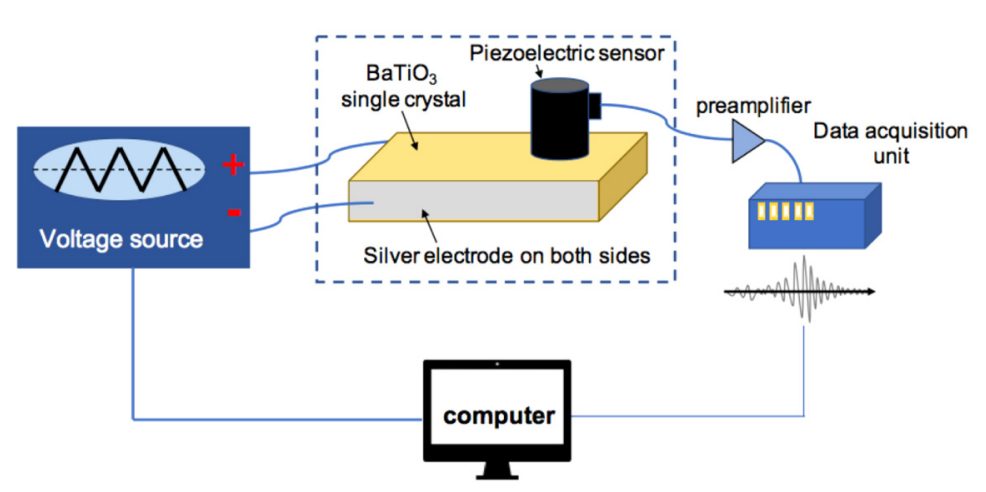

FIG. 4. Experimental arrangement for measuring acoustic emission (AE) during ferroelectric switching. The sample is a thin slab with $\sim 3 \mathrm{~mm}$ thickness and $10 \mathrm{~mm}^{2}$ surface area. The side surfaces of the sample are conducting. The switching field is applied to the conducting surfaces, and the rate is extremely low to allow the sample to relax during the switching process. The AE signal is measured by one or several piezoelectric strain wave detectors, preamplified and analyzed using calibrated circuits. This allows the determination of the absolute energy of the acoustic signals in attojoules $\left(10^{-18} \mathrm{~J}\right)$. After Salje et al., Phys. Rev. Mater. 3, 014415 (2019). Copyright 2019 American Physical Society. 


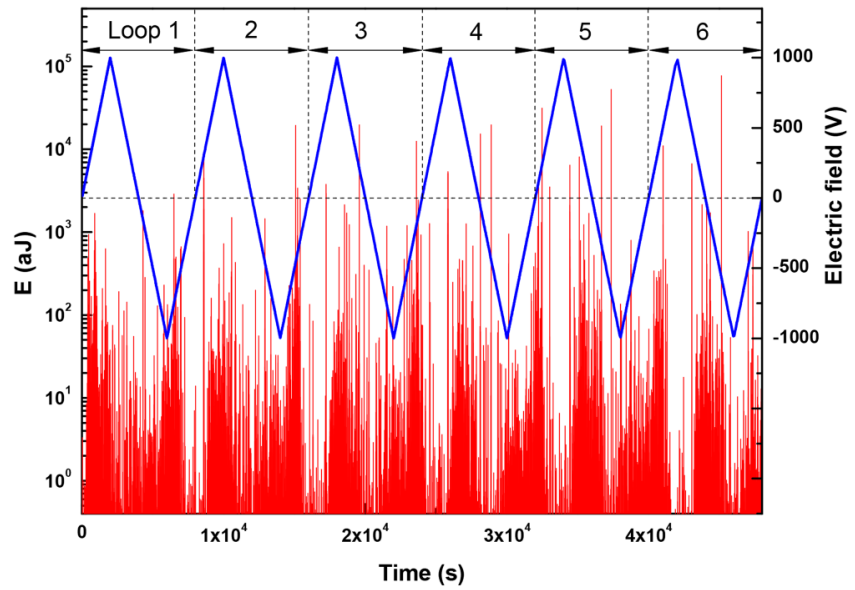

FIG. 5. Acoustic emission (AE) spectra of $\mathrm{BaTiO}_{3}$ at room temperature. The blue line indicates the extremely slow time evolution of the applied electric voltage between $-1000 \mathrm{~V}$ and $+1000 \mathrm{~V}$, the red peaks are the AE signals ( jerks) due to switching domains in units of attojoules. After Salje et al., Phys. Rev. Mater. 3, 014415 (2019). Copyright 2019 American Physical Society.

(Casals et al., 2019) and confirmed the statistical analysis (Casals et al., 2020).

More recently, the change of the fractal dimension of the domain patterns was measured (Xu et al., 2020) and a clear anticorrelation between the fractal Hausdorff dimension and the energy/amplitude exponents were found. The approach to criticality near the coercive field, thus, reduces the energy exponents and increases the fractal dimension. This shows that domain movements do not keep the pattern invariant by simply changing the position of length scale of the domain pattern, but topologically change the pattern with nucleation of switched island, domain dendrites, etc., which act as transient states during switching.

\section{SOME POSSIBLE DEVICE DESIGN PATHWAYS}

Ferroelastic domain walls can themselves either be the active element in a device or can couple with other ferroic materials, e.g., in thin cover layers. The former includes ferroelectric switching for memory devices, which we shortly review as a typical example. The direct switching of polar vectors in a twin wall is not yet experimentally proven, although it was found in computer simulations of cryogenic $\mathrm{SrTiO}_{3}$ (Zykova-Timan and Salje, 2014), while other findings seem to indicate a very limited scope for switching (Schiaffino and Stengel, 2018). Switching is even more likely in junctions between twin walls. These areas of domain wall crossings can develop much stronger dipolar moments, and these dipoles contain biquadratic coupling with the polarization. This coupling, in contrast to gradient coupling, allows polarization reversal under external fields. First experimental evidence was found at room temperature in $\mathrm{LaAlO}_{3}$ with a tweed nano-structure and a high junction density. These ferroelastic crystals show strong piezoelectric and polar responses and appear to indicate, at least, partial switching (Yokota et al., 2018; 2020; Kustov et al., 2018; and
Salje et al., 2016). An alternative pathway follows the secondary magnetic properties of ferroelastic needle domains. Ferroelastic vortex structures near moving needles contain displacement currents that generate magnetic dipoles on either side of the domain (Lu et al., 2020). This allows designing a device where the needle domain position is driven elastically or electrically. The readout is then magnetic at sites where the dynamic needle tip is located. Each memory element could then contain either one needle or a needle comb where the length of the needle is modified by external fields. The magnetic sensor would then be a magnetic quantum dot near the needle or a magnetic AFM tip. This configuration is then close to the idea of Parkin et al. (2008).

The second technique is to cover the ferroelastic film either by a high current layer of $\mathrm{SrTiO}_{3} / \mathrm{LaAlO}_{3}$ as shown by Frenkel et al. (2017). In this case, electronic properties, such as conductivity and superconductivity, can be tuned by the ferroelastic domain walls
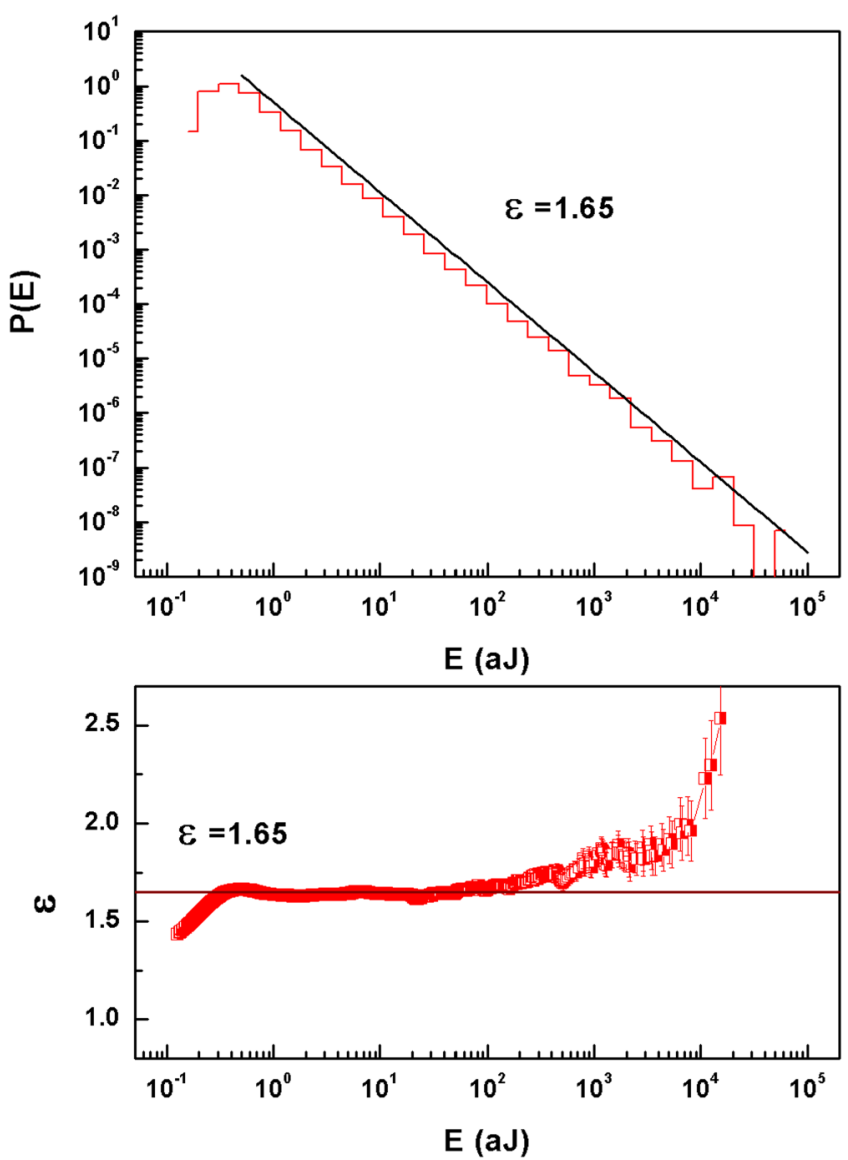

FIG. 6. Power law distribution of the jerk energies of the acoustic emission in $\mathrm{BaTiO}_{3}$. The upper curves shows the probability distribution $P(E)$ in log-log representation with an power law exponent $\epsilon=1.65$. A very robust analysis using the maximum-likelihood method (Baró and Vives, 2012 and Salje et al., 2019) shows the same exponent as a plateau of the ML curve. After Salje et al., Phys. Rev. Mater. 3, 014415 (2019). Copyright 2019 American Physical Society. 
and then used to create and control circuit elements and gatedefined devices. They showed that naturally occurring twin boundaries determine the $\mathrm{LaAlO}_{3} / \mathrm{SrTiO}_{3}$ interface $2 \mathrm{DEG}$ at the nanoscale. Polar $\mathrm{SrTiO}_{3}$ domain boundaries remain highly mobile down to very low temperatures. When localized pressure is applied to an individual $\mathrm{SrTiO}_{3}$ twin boundary, it leads to a change in $\mathrm{LaAlO}_{3} / \mathrm{SrTiO}_{3}$ interface current distribution. The existence of polarity at the twin boundaries make the ferroelastic twin boundaries serve as effective tunable gates.

\section{CONCLUSION}

Mobile ferroelastic walls carry their emerging processes with them during their movements. A key ingredient for applications in domain boundary engineering is hence to develop concepts to capture the exact nature of the movements. With increasing knowledge of the finer details of these motions, the great complexity of domain wall dynamics became clearer. The careful distinction between smooth and non-smooth (mild and wild) behavior has led to the discovery of domain wall motion by avalanches. These avalanches change the fractal dimension of the domain patterns and transfer much of the energy during the switching process. They follow, as far as we know today, mainly mean field behavior, while their analysis is still at a very early stage of development.

\section{ACKNOWLEDGMENTS}

The author is grateful to EPSRC for support (Grant No. EP/ K009702/1).

\section{DATA AVAILABILITY}

The data that support the findings of this study are available from the corresponding author upon reasonable request.

\section{REFERENCES}

Aird, A. and Salje, E. K. H., "Sheet superconductivity in twin walls: Experimental evidence of $\mathrm{WO}_{3-\mathrm{x}}$, J. Phys. Condens. Matter 10(22), L377 (1998).

Alava, M. J., Nukala, P. K. V. V., and Zapperi, S., "Statistical models of fracture," Adv. Phys. 55, 349 (2006).

Baró, J. and Vives, E., “Analysis of power-law exponents by maximum-likelihood maps,” Phys. Rev. E 85, 066121 (2012).

Carpenter, M. A., "Static and dynamic strain coupling behaviour of ferroic and multiferroic perovskites from resonant ultrasound spectroscopy," J. Phys. Condens. Matter 27, 263201 (2015).

Casals, B., Nataf, G. F., Pesquera, D., and Salje, E. K. H., "Avalanches from charged domain wall motion in $\mathrm{BaTiO}_{3}$ during ferroelectric switching," APL Mater. 8, 011105 (2020).

Casals, B., Nataf, G. F., and Salje, E. K. H., "Avalanche criticality during ferroelectric switching" (unpublished).

Casals, B., van Dijken, S., Herranz, G., and Salje, E. K. H., "Electric-field-induced avalanches and glassiness of mobile ferroelastic twin domains in cryogenic $\mathrm{SrTiO}_{3}$," Phys. Rev. Res. 1, 032025(R) (2019).

Catalan, G., Seidel, J., Ramesh, R., and Scott, J. F., "Domain wall nanoelectronics," Rev. Mod. Phys. 84, 119-156 (2012).

Chen, Y., Wang, Q., Ding, X., Sun J., and Salje, E. K. H., “Avalanches and mixing behavior of porous 316L stainless steel under tension,” Appl. Phys. Lett. 116, 111901 (2020).
Christensen, D. V., Frenkel, Y., Chen, Y. Z., Xie, Y. W., Chen, Z. Y., Hikita, Y., Smith, A., Klein, L., Hwand, H. Y., Pryds, N., and Kalinsky, B., "Strain-tunable magnetism at oxide domain walls," Nat. Phys. 15, 269-274 (2019).

Chrosch, L. and Salje, E. K. H., "Temperature dependence of the domain wall width in $\mathrm{LaAlO}_{3}$," J. Appl. Phys. 85, 722-727 (1999).

Clauset, A., Shalizi, C. R., and Newman, M. E. J., "Power-law distributions in empirical data," SIAM Rev. 51, 661 (2009).

Conti, S., Muller, S., Poliakovsky, A., and Salje, E. K. H., "Coupling of order parameters, chirality, and interfacial structures in multiferroic materials," J. Phys. Condens. Matter 23, 142203 (2011).

Emelyanov, A. Y., Pertsev, N. A., and Salje, E. K. H., "Effect of finite domain-wall width on the domain structures of epitaxial ferroelectric and ferroelastic thin films," J. Appl. Phys. 89, 1355-1366 (2001).

Eng, L. M., Guntherodt, H. J., Schneider, G. A., Kopke, U., and Saldana, J. M., "Nanoscale reconstruction of surface crystallography from three-dimensional polarization distribution in ferroelectric barium-titanate ceramics," Appl. Phys. Lett. 74, 233-235 (1999).

Farokhipoor, S. and Noheda, B., "Conduction through $71^{\circ}$ domain walls in $\mathrm{BiFeO}_{3}$ thin films,” Phys. Rev. Lett. 107(12), 127601 (2011).

Fontcuberta, J., Skumryev, V., Laukhin, V., Granados, X., and Salje, E. K. H., "Polar domain walls trigger magnetoelectric coupling," Sci. Rep. 5, 13784 (2015).

Frenkel, T., Haham, N., Shperber, Y., Bell, C., Xie, Y., Chen, Z., Hikita, Y., Hwang, H. Y., Salje, E. K. H., and Kalinsky, B., "Imaging and tuning polarity at $\mathrm{SrTiO}_{3}$ domain walls," Nat. Mater. 16, 1203 (2017).

Friedman, N., Jennings, A., Tsekenis, G., Kim, J. Y., Tao, M. L., Uhl, J. T., Greer, J. R., and Dahmen, K. A., "Statistics of dislocation slip avalanches in nanosized single crystals show tuned critical behavior predicted by a simple mean field model," Phys. Rev. Lett. 109, 095507 (2012).

Gallardo, M. C., Manchado, J., Romero, F. J., del Cerro, J., Salje, E. K. H., Planes, A., Vives, E., Romero, R., and Stipcich, M., "Avalanche criticality in the martensitic transition of $\mathrm{Cu}_{67.64} \mathrm{Zn}_{16.71} \mathrm{Al}_{15.65}$ shape-memory alloy: A calorimetric and acoustic emission study," Phys. Rev. B 81, 174102 (2010).

Goncalves-Ferreira, L., Redfern, S. A. T., Artacho, E., and Salje E. K. H., "Ferrielectric twin walls in $\mathrm{CaTiO}_{3}$," Phys. Rev. Lett. 101(9), 097602 (2008).

Gonnissen, J., Batuk, D., Nataf, G., Jones, G. F. L., Abakumov, A. M., Van Aert, S., Schryvers, D., and Salje, E. K. H., "Direct observation of ferroelectric domain walls in $\mathrm{LiNbO}_{3}$ : Wall-meanders, kinks, and local electric charges," Adv. Funct. Mater. 26, 7599-7604 (2016).

Gumbsch, P. and Gao, H., "Dislocations faster than the speed of sound," Science 283, 965-968 (1999).

Guyonnet, J. et al., "Conduction at domain walls in insulating $\mathrm{Pb}\left(\mathrm{Zr}_{0.2} \mathrm{Ti}_{0.8}\right) \mathrm{O}_{3}$ thin films," Adv. Mater. 23(45), 5377 (2011).

Harrison, R. and Salje, E. K. H., "The noise of the needle: Avalanches of a single progressing needle domain in $\mathrm{LaAlO}_{3}$," Appl. Phys. Lett. 97, 021907 (2010).

Harrison, R. and Salje, E. K. H., "Ferroic switching, avalanches, and the Larkin length: Needle domains in $\mathrm{LaAlO}_{3}$," Appl. Phys. Lett. 99, 151915 (2011).

Hayward, S. A., Chrosch, J., Salje, E. K. H., and Carpenter, M. A., “Thickness of pericline walls in anorthoclase: An x-ray diffraction," Eur. J. Miner. 8, 1301-1310 (1996).

Hayward, S. A., Morrison, F. D., Redfern, S. A. T., Salje, E. K. H., Scott, J. F., Knight, K. S., Tarantino, S., Glazer, A. M., Shuvaeva, V., Daniel, P., Zhang, M., and Carpenter, M. A., "Transformation processes in $\mathrm{LaAlO}_{3}$ : Neutron diffraction, dielectric, thermal, optical, and Raman studies," Phys. Rev. B 72, 054110 (2005).

Ishibashi, Y. and Takagi, Y., "Note on ferroelectric domain switching," J. Phys. Soc. Jpn. 31(2), 506 (1971).

Janovec, V., Richterová, L., and Pívratská, J., "Polar properties of compatible ferroelastic domain walls," Ferroelectrics 222(1), 73 (1999).

Jiang, J., Bai, Z. L., Chen, Z. H., He, L., Zhang, D. W., Zhang, Q. H., Shi, J. A., Park, M. H., Scott, J. F., Hwang, C. S., and Jiang, A. Q., "Temporary formation of highly conducting domain walls for non-destructive read-out of 
ferroelectric domain-wall resistance switching memories," Nat. Mater. 17, 49 (2018).

Jin, Z. H., Gumbsch, P., Ma, E., Albe, K., Lu, K., Hahn, H., and Gleiter, H., "The interaction mechanism of screw dislocations with coherent twin boundaries in different face-centred cubic metals," Scr. Mater. 54, 1163-1168 (2006).

Kim, Y., Alexe, M., and Salje, E. K. H., "Nanoscale properties of thin twin walls and surface layers in piezoelectric $\mathrm{WO}_{3-\mathrm{x}}$ ” Appl. Phys. Lett. 96, 032904 (2010).

Kirbus, B., Godau, C., Wehmeier, L., Beyreuther, E., Haussmann, A., and Eng, L. M., "Real-time 3D imaging of nanoscale ferroelectric domain wall dynamics in lithium niobate single crystals under electric stimuli: Implications for domain-wall-based nanoelectronic devices," ACS Appl. Nano Mater. 2, 5787-5794 (2019).

Kustov, S., Liubimova, I., and Salje, E. K. H., "LaAlO unusual ferroelastic properties,” Appl. Phys. Lett. 112, 042902 (2018).

Lawless, W. N. and Fousek, J., "Small-signal permittivity of the stationary (100)- $180^{\circ}$ domain wall in $\mathrm{BaTiO}_{3}$," J. Phys. Soc. Jpn. 28(2), 419 (1970).

Lee, W. T., Salje, E. K. H., Goncalves-Ferreira, L., Daraktchiev, M., and Bismayer, U., "Intrinsic activation energy for twin-wall motion in the ferroelastic perovskite $\mathrm{CaTiO}_{3}$," Phys. Rev. B 73, 214110 (2006).

Lu, G., Li, S., Ding, X., Sun, J., and Salje, E. K. H., "Electrically driven ferroelastic domain walls, domain wall interactions, and moving needle domains," Phys. Rev. Mater. 3, 114405 (2019a).

Lu, G., Li, S., Ding, X., Sun, J., and Salje, E. K. H., "Piezoelectricity and electrostriction in ferroelastic materials with polar twin boundaries and domain junctions," Appl. Phys. Lett. 114, 202901 (2019b).

Lu, G., Li, S., Ding, X., Sun, J., and Salje, E. K. H., "Current vortices and magnetic fields driven by moving polar twin boundaries in ferroelastic materials," NPJ Comput. Mater. 6, 1-6 (2020).

Ma, J., Ma, J., Zhang, Q., Peng, R., Wang, J., Liu, C., Wang, M., Li, N., Chen, M., Cheng, X., Gao, P., Gu, L., Chen, L.-Q., Yu, P., Zhang, J., and Nan, C.-W., "Controllable conductive readout in self-assembled, topologically confined ferroelectric domain walls," Nat. Nanotechnol. 13(10), 947 (2018).

Meier, D., "Functional domain walls in multiferroics," J. Phys. Condens. Matter 27(46), 463003 (2015).

Meier, D. et al., "Anisotropic conductance at improper ferroelectric domain walls," Nat. Mater. 11(4), 284 (2012).

Miller, R. C., "Some experiments on the motion of $180^{\circ}$ domain walls in $\mathrm{BaTiO}_{3}$," Phys. Rev. 111(3), 736 (1958).

Nataf, G., Castillo-Villa, P. O., Boro, J., Illia, X., Vives, E., Planes, A., and Salje, E. K. H., "Avalanches in compressed porous $\mathrm{SiO}_{2}$-based materials," Phys. Rev. E 90, 022405 (2014)

Nataf, G. F., Guennou, M., Kreisel, J., Hicher, P., Haumont, R., Aktas, O., Salje, E. K. H., Tortech, L., Mathieu, C., Martinotti, D., and Barrett, N., "Control of surface potential at polar domain walls in a nonpolar oxide," Phys. Rev. Mater. 1, 074410 (2017).

Nataf, G. F. and Guennou, M., "Optical studies of ferroelectric and ferroelastic domain walls," J. Phys. Condens. Matter 32(18), 183001 (2020).

Parkin, S. P. P., Hayashi, M., and Thomas, L., "Magnetic domain-wall racetrack memory," Science 320, 190-194 (2008).

Pertsev, N. A., Novak, J., and Salje, E. K. H., "Long-range elastic interactions and equilibrium shapes of curved ferroelastic domain walls in crystals," Philos. Mag. A 80, 2201-2213 (2000).

Puchberger, S., Soprunyuk, V., Schranz, W., Troster, A., Roleder, K., Majchrowski, A., Carpenter, M. A., and Salje, E. K. H., "The noise of many needles: Jerky domain wall propagation in $\mathrm{PbZrO}_{3}$ and $\mathrm{LaAlO}_{3}$," Appl. Phys. Lett. Mater. 5, 046102 (2017).

Rojac, T. et al., "Domain-wall conduction in ferroelectric $\mathrm{BiFeO}_{3}$ controlled by accumulation of charged defects," Nat. Mater. 16(3), 322 (2017).

Salje, E., "Kinetic rate laws as derived from order parameter theory. 1. Theoretical concepts," Phys. Chem. Miner. 15, 336-348 (1988).

Salje, E. K. H., "Hard mode spectroscopy-Experimental studies of structural phase transitions," Phase Transitions 37, 83-110 (1992).

Salje, E. K. H., Phase Transitions in Ferroelastic and Co-elastic Crystals (Cambridge University Press, 1993).
Salje, E. K. H., "Multiferroic domain boundaries as active memory devices: Trajectories towards domain boundary engineering," CHEMPHYSCHEM 11, 940-950 (2010).

Salje, E. K. H., "Ferroelastic materials," Annu. Rev. Mater. Res. 42, 265-283 (2012).

Salje, E. K. H. and Dahmen, K. A., "Crackling noise in disordered materials," Annu. Rev. Condens. Matter Phys. 5(1), 233 (2014).

Salje, E. K. H. and Ishibashi, Y., "Mesoscopic structures in ferroelastic crystals: Needle twins and right-angled domains," J. Phys. Condens. Matter 8, 8477-8495 (1996).

Salje, E. and Parlinski, K., "Microstructures in high $\mathrm{T}_{c}$ superconductors," Supercond. Sci. Technol. 4, 93-97 (1991).

Salje, E. and Wruck, B., "Specific-heat measurements and critical exponents of the ferroelastic phase-transition in $\mathrm{Pb}_{3}\left(\mathrm{PO}_{3}\right)_{2}$ and $\mathrm{Pb}_{3}\left(\mathrm{P}_{1-\mathrm{x}} \mathrm{As}_{\mathrm{x}} \mathrm{O}_{4}\right)_{2}$," Phys. Rev. B 28, 6510-6518 (1983).

Salje, E. K. H., Alexe, M., and Kustov, S., "Direct observation of polar tweed in $\mathrm{LaAlO}_{3}$," Sci. Rep. 6, 27193 (2016).

Salje, E., Palosz, B., and Wruck, B., "In-situ observation of the polytypic phasetransition $2 \mathrm{H}-12 \mathrm{R}$ in $\mathrm{PbI}_{2}$-Investigation of the thermodynamic, structural and dielectric properties," J. Phys. C 20, 4077-4096 (1987).

Salje, E. K. H., Planes, A., and Vives, E., "Analysis of crackling noise using the maximumlikelihood method: Power-law mixing and exponential damping," Phys. Rev. E 96, 042122 (2017a).

Salje, E. K. H., Aktas, O., Carpenter, M. A., and Scott, J. F., "Domains within domains and walls within walls: Evidence for polar domains in cryogenic $\mathrm{SrTiO}_{3}$," Phys. Rev. Lett. 111, 247603 (2013)

Salje, E. K. H., Buckley, A., Van Tendeloo, G., and Ishibashi, Y., "Needle twins and right-angled twins in minerals: Comparison between experiment and theory," Am. Miner. 83, 811-822 (1998).

Salje, E., Devarajan, V., Bismayer, U., and Guimaraes, D. M. C., "Phase transitions in $\mathrm{Pb}_{3}\left(\mathrm{PO}_{4}\right)_{2}$-Influence of the central peak and flip mode on the Raman scattering of hard modes," J. Phys. C Solid State Phys. 16, 5233-5243 (1983).

Salje, E. K. H., Wang, X., Ding, X., and Scott, J. F., "Ultrafast switching in avalanche-driven ferroelectrics by supersonic kink movements," Adv. Funct. Mater. 27, 1700367 (2017b)

Salje, E. K. H., Xue, D., Ding, X., Dahmen, K. A., and Scott, J. F., "Ferroelectric switching and scale invariant avalanches in $\mathrm{BaTiO}_{3}$," Phys. Rev. Mater. 3, 014415 (2019).

Salje, E. K. H., Safarik, D. J., Modic, K. A., Gubernatis, J. E., Cooley, J. C., Taylor, R. D., Mihaila, B., Saxena, A., Lookman, T., Smith, J. L., Fisher, R. A., Pasternak, M., Opeil, C. P., Siegrist, T., Littlewood, P. B., and Lashley, J. C., "Tin tellurite; a weakly co-elastic metal," Phys. Rev. B 82, 184112 (2010).

Schiaffino, A. and Stengel, M., "Macroscopic polarization from antiferrodistortive cycloids in ferroelastic $\mathrm{SrTiO}_{3}$," Phys. Rev. Lett. 119, 137601 (2018).

Scott, J. F., Salje, E. K. H., and Carpenter, M. A., "Domain wall damping and elastic softening in $\mathrm{SrTiO}_{3}$ : Evidence for polar twin walls," Phys. Rev. Lett. 109, 187601 (2012).

Seidel, J., "Domain walls as nanoscale functional elements," J. Phys. Chem. Lett. 3, 2905 (2012)

Seidel, J. et al., "Conduction at domain walls in oxide multiferroics," Nat. Mater. 8(3), 229 (2009).

Seidel, J. et al., "Domain wall conductivity in La-doped $\mathrm{BiFeO}_{3}$," Phys. Rev. Lett. 105(19), 197603 (2010)

Sethna, J. P., Dahmen, K. A., and Myers, C. R., "Crackling noise," Nature 410(6825), 242 (2001).

Shilo, D., Ravichandran, G., and Bhattacharya, K., "Investigation of twin-wall structure at the nanometre scale using atomic force microscopy," Nat. Mater. 3, 453-457 (2004)

Sluka, T. et al., "Free-electron gas at charged domain walls in insulating $\mathrm{BaTiO}_{3}$," Nat. Commun. 4, 1808 (2013).

Van Aert, S., Turner, S., Delville, R., Schryvers, D., Van Tendeloo, G., and Salje, E. K. H., "Direct observation of ferrielectricity at ferroelastic domain boundaries in $\mathrm{CaTiO}_{3}$ by electron microscopy," Adv. Mater. 24, 523 (2012). 
Viehland, D. D. and Salje, E. K. H., "Domain boundary-dominated systems: Adaptive structures and functional twin boundaries," Adv. Phys. 63, 267-326 (2014).

Vives, E., Ortín, J., Mañosa, L., Ràfols, I., Pérez-Magrané, R., and Planes, A., "Distributions of avalanches in martensitic transformations," Phys. Rev. Lett. 72, 1694-1697 (1994).

Wei, X.-K., Tagantsex, A. K., Kvasov, A., Roleder, K., Jia, C.-L., and Setter, N. "Ferroelectric translational antiphase boundaries in nonpolar materials," Nat. Commun. 5, 3031 (2014).

Weiss, J., Ben Rhouma, W., Richeton, T., Dechanel, S., Louchet, F., and Truskinovsky, L., "From mild to wild fluctuations in crystal plasticity," Phys. Rev. Lett. 114, 105504 (2015).

Xu, Y., Zhou, Y., Xue, D., Ding, X., Sun, J., and Salje, E. K. H., “Anisotropic avalanche dynamics during ferroelectic switching in $\mathrm{BaTiO}_{3}$ and $0.7 \mathrm{~Pb}\left(\mathrm{Mg}_{2 / 3} \mathrm{Nb}_{1 / 3}\right) \mathrm{O}_{3}-0.3 \mathrm{PbTiO}_{3}$," Appl. Phys. Lett. (to be published) (2020).

Yang, M.-M. and Alexe, M., "Photoelectric effects at domain walls," in Domain Walls From Fundamental Properties to Nanotechnology Concepts, edited by D. Meier, J. Seidel, M. Gregg, and R. Ramesh (Oxford University Press, Oxford, 2020).

Yang, Y., Li, S., Ding, X., and Salje, E. K. H., "Twisting of pre-twinned alpha-Fe nanowires: From mild to wild avalanche dynamics," Acta Mater. 195, 50-58 (2020).

Yang, Y., Li, S., Ding, X., Sun, J., and Salje, E. K. H., "Interface driven pseudo-elasticity in a-Fe nanowires," Adv. Funct. Mater. 26, 760-767 (2016).

Yokota, H., Matsumoto, S., Salje, E. K. H., and Uesu, Y., "Symmetry and threedimensional anisotropy of polar domain boundaries observed in ferroelastic $\mathrm{LaAlO}_{3}$ in the complete absence of ferroelectric instability," Phys. Rev. B 98, 104105 (2018).
Yokota, H., Matsumoto, S., Salje, E. K. H., and Uesu, Y., "Polar nature of domain boundaries in purely ferroelastic $\mathrm{Pb}_{3}\left(\mathrm{PO}_{4}\right)_{2}$ investigated by second harmonic generation microscopy," Phys. Rev. B 100, 024101 (2019).

Yokota, H., Hasegawa, N., Glazer, M., Salje, E. K. H., and Uesu, Y., "Direct evidence of polar ferroelastic domain boundaries in semiconductor $\mathrm{BiVO}_{4}$," Appl. Phys. Lett. 116, 232901 (2020b).

Yokota, H., Matsumoto, S., Hasegawa, N., Salje, E. K. H., and Uesu, Y., "Enhancement of polar nature of domain boundaries in ferroelastic $\mathrm{Pb}_{3}\left(\mathrm{PO}_{4}\right)_{2}$ by doping divalent-metal ions," J. Phys. Condens. Matter 32, 345401 (2020a).

Yokota, H., Niki, S., Haumont, R., Hicher, P., and Uesu, Y., "Polar nature of stressinduced twin walls in ferroelastic $\mathrm{CaTiO}_{3}$," AIP Adv. 7(8), 085315 (2017).

Yokota, H., Usami, H., Haumont, R., Hicher, P., Kaneshiro, J., Salje, E. K. H., and Uesu, Y., "Direct evidence of polar nature of ferroelastic twin boundaries in $\mathrm{CaTiO}_{3}$ obtained by second harmonic generation microscope," Phys. Rev. B 89, 144109 (2014).

Yokota, H., Haines, C. R. S., Matsumoto, S., Hasegawa, N., Carpenter, M. A., Heo, Y., Marin, A., Salje, E. K. H., and Uesu, Y., "Domain wall generated polarity in ferroelastics: Results from resonance piezoelectric spectroscopy, piezoelectric force microscopy, and optical second harmonic generation measurements in $\mathrm{LaAlO}_{3}$," Phys. Rev. B 102, 104117 (2020).

Zaiser, M., "Scale invariance in plastic flow of crystalline solids," Adv. Phys. 55(1-2), 185-245 (2006).

Zhang, L., Li, S., Ding, X., Sun, J., and Salje, E. K. H., "Statistical analysis of emission, interaction and annihilation of phonons by kink motion in ferroelastic materials," Appl. Phys. Lett. 116, 102902 (2020).

Zykova-Timan, T. and Salje, E. K. H., "Highly mobile vortex structures inside polar twin boundaries in $\mathrm{SrTiO}_{3}$," Appl. Phys. Lett. 104, 082907 (2014). 